Nouvelles perspectives en sciences sociales

Revue internationale de systémique complexe et d'études relationnelles

\title{
Les simulations computationnelles dans les sciences sociales
}

\section{Franck Varenne}

Volume 5, numéro 2, mai 2010

Sur le thème de la simulation

URI : https://id.erudit.org/iderudit/044073ar

DOI : https://doi.org/10.7202/044073ar

Aller au sommaire du numéro

\section{Éditeur(s)}

Prise de parole

\section{ISSN}

1712-8307 (imprimé)

1918-7475 (numérique)

Découvrir la revue

\section{Citer cet article}

Varenne, F. (2010). Les simulations computationnelles dans les sciences sociales. Nouvelles perspectives en sciences sociales, 5(2), 17-49. https://doi.org/10.7202/044073ar

\section{Résumé de l'article}

Les sciences sociales entrent résolument - quoique partiellement - dans l'ère computationnelle. Ce constat n'a pas encore de sens précis si on ne l'accompagne d'une analyse discriminante des fonctions épistémiques de la computation dans les différents recours aux ordinateurs pour la modélisation et la simulation en sciences sociales. De par l'introduction de ces nouvelles manières de formaliser (séduisantes car apparaissant comme plus directes et plus ergonomiques), la double question du réalisme des formalismes et de la valeur de preuve des traitements computationnels se pose à nouveaux frais. Cette expansion tous azimuts des simulations computationnelles conduit certains observateurs enthousiastes à penser que l'on a là un nouveau fondement commun pour toutes les sciences sociales. En clarifiant et en distinguant certains des usages épistémiques de différentes simulations computationnelles dans les sciences sociales, cet article montre cependant qu'il vaut mieux s'en tenir à une position médiane et soutenir que l'apport en est principalement méthodologique. 


\title{
Les simulations computationnelles dans les sciences sociales
}

\author{
Franck VarenNe \\ Université de Rouen \& GEMAS
}

\section{Introduction : computation et sciences computationnelles}

A partir des années 1980 pour les sciences physiques, la chimie

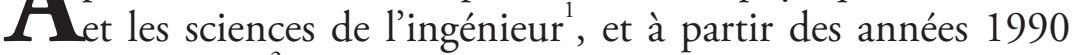
pour la biologie ${ }^{2}$, on a assisté à une série de tournants computationnels, c'est-à-dire à une série d'extensions considérables du recours à l'ordinateur dans les pratiques de modélisation. On pourrait objecter que la physique et la chimie recourraient déjà à l'ordinateur dès sa conception et sa diffusion, c'est-à-dire dès les années 1940-1950. Il ne faudrait pas confondre cependant ce qui a été majoritairement et immédiatement un usage mathématique de l'ordinateur avec ce qui est devenu plus tardivement un

$1 \quad$ Voir par exemple Modélisation mathématique et simulation numérique, Les Ulis, EDP Sciences, 1999. Voir également une première réflexion épistémologique (d'inspiration classique) de Paul Humphreys dans Extending Ourselves: Computational Science, Empiricism and Scientific Method, Oxford, Oxford University Press, 2004.

2 Voir Marek Michalewicz, Plants to Ecosystems. Advances in Computational Life Sciences, Collingwood (Australia), CSIRO Publishing, 1997; FranckVarenne, "La simulation informatique face à la méthode des modèles ", Natures Sciences Sociétés, vol 11, $\mathrm{n}^{\circ}$ 1, 2003, p. 16-28; Franck Varenne, Du modèle à la simulation informatique, Paris, Vrin, 2007. 
usage computationnel de l'ordinateur. Un usage mathématique de l'ordinateur est un usage qui l'institue comme machine de calcul et de résolution numérique approchée (mathématique en ce sens étroit) d'un modèle mathématique préalablement formulé et analytiquement non soluble. Dans cette perspective, le physicien numéricien peut écrire :

Un modèle mathématique est une représentation ou une interprétation abstraite de la réalité physique qui est accessible à l'analyse et au calcul. La simulation numérique permet de calculer sur ordinateur les solutions de ces modèles, et donc de simuler la réalité physique ${ }^{3}$.

Par contraste, on peut définir un usage computationnel comme ce qui fait de l'ordinateur une machine à modéliser au moyen de computations. Une computation est une opération pas à pas sur des symboles. Elle peut elle-même être interprétée comme un calcul de modèle ou comme la simulation directe du comportement d'un système cible (réel ou fictif). Mais cela ne lui est nullement nécessaire. Au sens le plus général, une science computationnelle est une science qui utilise l'ordinateur comme une machine à modéliser et à simuler au moyen de computations. Je reviendrai sur la polysémie du terme "simulation " telle qu'elle apparaît alors dans cette caractérisation.

Rappelons que, depuis la fin des années 1990, les modèles computationnels se sont également largement diffusés dans les sciences sociales : je renvoie à ce sujet aux contributions importantes de Epstein, d'Axtell, de Gilbert et Troitzsch, ou de Conte citées en bibliographie, ainsi qu'au tour d'horizon de Heath et al. ${ }^{4}$ Or, au sujet de l'interprétation épistémologique à donner à ce tournant computationnel actuellement négocié par une frange

3 Grégoire Allaire, Analyse numérique et optimisation, Paris, Éditions de l'École Polytechnique, 2007. Je montrerai plus bas que cette description rapide (notamment avec l'affirmation déductive associée « et donc de simuler la réalité physique » qui, à certains égards, commet une erreur de catégorie), ne peut valoir en toute généralité pour tout type de simulation sur ordinateur. C'est a fortiori le cas pour les simulations en sciences sociales.

4 Brian Heath, Raymond Hill et Frank Ciarallo, "A Survey of Agent-Based Modeling Practices (January 1998 to July 2008) ", Journal of Artificial Societies and Social Simulation, vol. 12, n 4, 2009, http://jasss.soc.surrey.ac.uk/12/4/9. html. 
croissante des spécialistes en sciences sociales, on trouve grosso modo trois thèses qui s'affrontent dans les publications spécialisées, de la plus faible à la plus forte :

1- Thèse faible: l'adoption des simulations computationnelles s'interprète comme la simple diffusion d'un nouveau type de formalisme inspiré de la physique computationnelle et de l'intelligence artificielle distribuée.

2- Thèse moyenne : les simulations computationnelles proposent une nouvelle méthodologie aux sciences sociales, en permettant d'étendre le domaine de l'explication sociologique et d'entrer dans l'ère de l'" individualisme méthodologique complexe " appelée de ses vœux par Manzo ${ }^{5}$ et déjà annoncée en un sens par Dupuy ${ }^{6}$.

3- Thèse forte : au-delà d'une nouvelle méthodologie, il s'agit de rien de moins que d'une manière inédite de fonder une nouvelle unité des sciences sociales en dépassant les limites des méthodes de modélisation antérieures. Ainsi pensent par exemple Epstein et Axtell ${ }^{7}$.

Dans cet article, je ne trancherai pas directement. Cependant, je soutiendrai une série d'arguments qui plaident en faveur d'une thèse proche de la thèse moyenne. Mais avant d'en venir à cela, je partirai de l'hypothèse que, pour se faire une bonne idée du caractère innovant des simulations computationnelles en sciences sociales, il est bon d'abord de prendre la mesure de leur variété (sections 3 à 6), et pour cela, dans un premier temps de proposer des définitions et des distinctions conceptuelles qui permettent de donner du sens à cette variété (section 1) puis de comprendre précisément en quoi un modèle formel traité par ordinateur n'est pas systématiquement un modèle de simulation computationnelle (section 2).

5 Gianluca Manzo, «Progrès et "urgence” de la modélisation en sociologie. Du concept de "modèle générateur" et de sa mise en ouvre ", Année sociologie, vol. 57, n 1, 2007, p. 13-61.

6 Jean-Pierre Dupuy, Introduction aux sciences sociales, Paris, Ellipses, 1992.

7 Joshua Epstein et Robert Axtell, Growing Artificial Societies - Social Science from the Bottom Up, Cambridge, MIT Press, 1996. 
C'est seulement alors que la question du statut épistémique des divers types de simulations computationnelles en sciences sociales pourra être abordée (section 7) et que la question, plus générale et corrélative, de la nature de l'apport des méthodes computationnelles aux sciences sociales pourra enfin être plus clairement évoquée (conclusion).

\section{Modèles et simulations : distinctions conceptuelles}

Dans le contexte d'une recherche scientifique, un modèle se présente comme un objet, de nature symbolique ou matériel, qui, dans le cadre d'un questionnement, a pour fonction minimale de faciliter un accès cognitif à un objet cible via une médiation d'un type donné.

Pour cette définition, j'utilise une caractérisation déjà ancienne de Minsky ${ }^{8}$ que j'enrichis au moyen du constat empirique que l'on trouve dans l'ouvrage dirigé par Morgan et Morrison'. En se fondant sur cette définition, et en considérant ensuite l'espace de variation des types de médiation que le modèle peut faciliter, il est possible de distinguer vingt différentes fonctions épistémiques pour les modèles dans les sciences contemporaines ${ }^{10}$.

Sans entrer ici dans le détail de toutes ces fonctions, je rappellerai que l'on peut en distinguer cinq grandes catégories. Ces catégories sont ordonnées selon les types de tâches cognitives que le modèle facilite.

1- Il peut faciliter une expérience : un modèle peut servir à faciliter notre accès à ce qui se donne de manière sensible, mesurable ou détectable. Il facilite ainsi une observation, une visualisation, mais aussi le rendu d'une expérience ou d'une expérimentation.

2- Il peut faciliter une présentation ou une formulation

$8 \quad$ Marvin Minsky, "Matter, Mind and Models ", Proceedings of IFIP Congress, 1965, p. 45-49.

9 Mary Morgan et Margaret Morrison (dir.), Models as Mediators, Cambridge, Cambridge University Press, 1999.

10 Voir Franck Varenne, "Épistémologie des modèles et des simulations - Tour d'horizon et tendances ", dans Jean-Michel Lévy (dir.), Les modèles, possibilités et limites : jusqu'où va le réel?, Paris, EDP Sciences, à paraître en 2010. 
intelligible : un modèle peut faciliter notre accès intelligible à un objet cible grâce à une représentation mentale ou grâce à une conceptualisation de sa structure ou de sa dynamique. Cette conceptualisation peut elle-même prendre des formes variées, voire opposées. Elle peut prendre la forme de modèles phénoménologiques purement prédictifs ou de modèles à visée explicative, donc à scénario explicatif.

3- Il peut faciliter une théorisation : le modèle peut servir à élaborer progressivement une théorie non encore mature (essai localisé d'une structure d'inférence). Il peut servir aussi à illustrer une théorie, à interpréter une théorie, à tester la cohérence d'une théorie ou encore à en permettre l'applicabilité. Dans ce contexte, une théorie se distingue d'un modèle en ce qu'elle se présente comme un ensemble d'énoncés - éventuellement formalisés et axiomatisés - formant système et donnant lieu à des inférences susceptibles de valoir pour tout un type de phénomènes donné, alors qu'un modèle se limite souvent à mettre en ordre un ensemble de "contraintes particulières sur des interactions" ".

4- Il peut faciliter une médiation entre discours : un modèle peut être un instrument médiateur entre différentes disciplines, différents domaines du savoir ou différents groupes humains ayant des représentations collectives ou des intérêts divergents. Dans ce cadre-là, un modèle facilite une médiation non entre un objet et une appréhension directement sensible, instrumentée, conceptuelle ou théorique de cet objet, mais entre des discours et des représentations d'acteurs autour d'un problème ou d'un phénomène représenté de manière multiple quoique reconnu par toutes les parties en présence comme devant être abordé avec des questions communes. À ce titre, le modèle sert encore à un questionnement, mais il en reste à la première étape du questionnement. Il sert seulement à élaborer une formulation commune du questionnement plus qu'il ne permet une réponse à des

11 Selon l'expression de Pierre Livet : «Towards an Epistemology of Multi-Agent Simulations in Social Sciences ", dans Denis Phan et Frédéric Amblard (dir.), Agent-Based Modelling and Simulation in the Social and Human Sciences, Oxford, The Bardwell Press, GEMAS Studies in Social Analysis Series, 2007, p. 169-193. 
questions qui seraient déjà formulées de manière consensuelle.

5- Il peut faciliter une décision pour l'action (cognition pratique). Il existe ainsi des modèles qui ne servent pas tant à produire une représentation valable ni à formuler un questionnement qu'à faciliter la détermination d'un type de réponse attendu en termes d'action. Cette action est urgente et peut se passer de l'étape intermédiaire de représentation ou de conceptualisation en ce qu' elle vise à résoudre immédiatement un problème, par exemple, à diminuer des dommages imminents ou encore à optimiser des gains.

Qu'en est-il maintenant des simulations? De leur côté, elles ont longtemps été considérées comme n'étant qu'un certain type de modèles : des modèles phénoménologiques qui ne reproduisent que les performances, mais pas le mécanisme sous-jacent du système cible. Encore aujourd'hui, les simulations sont souvent réputées imiter un comportement visible ou mesurable, mais pas les processus ni les mécanismes à l'origine de ce comportement. On les range ainsi souvent parmi les modèles appartenant à la première catégorie : celle qui facilite un accès empirique ou quasi-empirique (par indirection et délégation) à l'objet cible.

Pourtant, on sait par ailleurs qu'avec l'essor de l'ordinateur et des traitements formels associés, le terme "simulation » a fini par désigner aussi le calcul d'un modèle. Est-ce par accident? Il y a certes des raisons historiques à cela ${ }^{12}$. Mais ces deux significations semblent aujourd'hui bien éloignées l'une de l'autre. Gardentelles toujours un rapport?

Ören $^{13}$ et Yilmaz ${ }^{14}$, par exemple, les présentent comme deux

12 Dans le domaine du calcul scientifique, les calculateurs numériques ont été précédés par des calculateurs analogiques (comme les analyseurs différentiels...) qu'on appelait déjà " simulateurs " parce qu'ils reproduisaient des performances visibles (en s'appuyant sur des analogies, voire des identités entre des systèmes d'équations de différents domaines de la physique) tout en ne reposant pas sur la même technologie que les systèmes cibles dont ils calculaient par avance le résultat.

13 Tüncer I. Ören, "Toward the Body of Knowledge of Modeling and Simulation ", Interservice/Industry Training, Simulation and Education Conference (I/ITSEC), Orlando, Florida, 2005, p. 1-19.

14 Levent Yilmaz, Tüncer I. Ören et Nasser Ghassem-Aghaee, "Intelligent agents, simulation, and gaming ", Simulation \& Gaming, vol. 37, $\mathrm{n}^{\circ} 3$, 
significations juxtaposées du même terme et, donc, comme n'entretenant plus de rapport essentiel, même indirect. Cependant, dans des publications précédentes ${ }^{15}$, j'ai montré que si l'on se donnait une définition plus générique du concept de simulation, les deux significations usuelles de ce terme pouvaient en dériver naturellement et qu'il en résultait une meilleure compréhension de leur rapport, puis une meilleure compréhension du rapport entre simulation et modèle.

De manière générale, une simulation peut se caractériser en effet comme une stratégie de symbolisation prenant la forme d'un traitement de symboles pas à pas, ce traitement pas à pas présentant lui-même deux phases distinctes :

Une première phase (dite opératoire) pendant laquelle une certaine quantité d'opérations s'effectue sur des entités symboliques numériquement distinctes. Ces entités sont considérées comme symboliques en ce qu'elles sont supposées dénoter des entités cibles (externes donc) réelles ou fictives. Les entités dénotées par ces symboles peuvent être des objets (réels ou fictifs), des parties ou des aspects d'objets ou de substrats, des individus, des acteurs, des agents, des représentations, des collectifs réifiés, des institutions...

Une seconde phase (dite observationnelle) pendant laquelle se produit une observation une mesure, une évaluation ou encore tout type d'analyse (par exemple statistique) ou de réemploi computationnel ou mathématique du résultat de la quantité d'opérations effectuée dans la première phase. Dans cette seconde phase, les résultats de computation sont considérés comme des données qu'il faut observer et analyser.

À la fin de la simulation, le résultat de la première phase symbolise lui aussi le système cible, mais à une autre échelle ou selon un autre point de vue. Du fait de ce traitement en deux phases, et par analogie avec le rapport qui existe entre le niveau

septembre 2006, p. 339-349.

15 Franck Varenne, Du modèle à la simulation informatique, op. cit.; Franck Varenne, "Modèles et simulations : pluriformaliser, simuler, remathématiser ", dans Jean-Jacques Kupiec et al. (dir.), Modèles, simulations, systèmes, Paris, Syllepse, 2008, p. 153-180. 
des paramètres d'un réseau de neurones et le niveau de la pensée symbolique - rapport dit de sous-symbolisation par Smolensky ${ }^{16}$-, les symboles élémentaires opérant dans la première phase d'une simulation peuvent être dits des sous-symboles relativement au symbole final.

Une simulation implique la constitution d'un symbole qui résulte d'opérations effectuées par des symboles élémentaires, pas nécessairement réalistes, mais dénotant à un autre niveau ou selon un autre point de vue que ce symbole résultant. Ces symboles opérant dans la première phase peuvent même adopter le point de vue de la fiction. C'est, par exemple, le cas pour les symboles intervenant dans la première phase d'une simulation dite "par éléments finis ", comme il en existe souvent en mécanique des fluides : ce sont des symboles dénotants sans dénotation, puisque, dans ce cas, les symboles d'éléments finis sont des trucs de calcul et non des symboles qui dénoteraient réellement des entités reconnues comme existantes et théorisées comme telles dans le domaine d'expertise considéré (ici, la physique des fluides).

\section{Pourquoi passer du modèle à la simulation en sciences sociales?}

Même si les sciences sociales ont développé une assez grande variété de pratiques de modélisation, les plus répandues au $\mathrm{XX}^{\mathrm{e}}$ siècle sont principalement de trois types.

1- Ce sont, d'abord, les modèles pour l'analyse de données. Ils appartiennent à la première grande catégorie, celle des modèles qui visent à faciliter une expérience ou la présentation de données de l'expérience. Au moyen de techniques comme la réduction de variance, ce type de modèle vise à repérer les corrélations, à résumer et à cribler l'information empirique. Il sert aussi à suggérer inductivement des liens causaux entre divers facteurs. Dès ses débuts, l'informatique a joué un rôle majeur dans le traitement numérique massif de données qu'impliquait ce type de modèle :

16 Paul Smolensky, "On the Proper Treatment of Connectionism ", The Behavioural and Brain Sciences, vol. 11, 1988, p. 1-74. 
mais précisément, cela n'engageait pas encore un usage computationnel de l'ordinateur.

2- Ce sont, ensuite, les modèles purement phénoménologiques et prédictifs, modèles souvent prônés par les économistes tenants d'un instrumentalisme positiviste à la Friedman (1953). Pour cette conception de la modélisation mathématique, un modèle sert à organiser de manière pratique et économe (selon le principe du rasoir d'Occam) le matériau empirique tout en permettant de faire des prédictions utiles.

3- Ce sont, enfin, les modèles explicatifs (ou modèles de mécanisme) qui se fondent sur des hypothèses théoriques, à validité plus ou moins locale, mais qui se veulent explicatives : grammaires génératives, modèles de l'acteur rationnel, modèles de préférences pour expliquer les phénomènes de ségrégation, structures d'interaction expliquant les stratégies scolaires... Ce genre de modèle permet de tester des hypothèses de mécanismes opérant au niveau de l'individu.

Remarquons que les deux derniers types de modèles appartiennent à la deuxième des catégories que j'ai distinguées plus haut, soit celle des modèles facilitant la formulation intelligible d'un phénomène, tantôt pour la prédiction, tantôt pour l'explication.

Dès les années 1960, Raymond Boudon ${ }^{17}$ a été l'un des promoteurs des modèles explicatifs en sociologie. Pour lui, il s'agissait d'aller au-delà des modèles statistiques ou des modèles phénoménologiques prédictifs et de faire œuvre de science en allant jusqu'au stade de l'explication. Or, dans la perspective de l'individualisme méthodologique qui était - et reste encore - la sienne, expliquer un phénomène social revient à le rapporter aux comportements des acteurs individuels. Cela impose donc de chercher à opérationnaliser, au niveau de l'individu, les hypothèses qu'une telle approche suggère; et cette opérationnalisation peut

17 Raymond Boudon, L'analyse mathématique des faits sociaux, Paris, Plon, 1967; Essais sur la théorie générale de la rationalité - Action sociale et sens commun, Paris, PUF, 2007; La rationalité, Paris, PUF, coll. "Que sais-je? ", 2009; avec Renaud Fillieule, Les méthodes en sociologie, Paris, PUF, coll. "Que sais-je? ", [1969] 2004. 
être prise en charge par des modèles de comportements des acteurs.

Par ailleurs, en sciences sociales, l'analyse de données bute souvent sur le phénomène d'interaction entre variables : l'intensité de l'effet qu'une variable a sur une autre dépend de l'intensité d'une troisième variable. Les variables ne sont pas seulement juxtaposées, elles se commandent les unes les autres. Dans ce cas-là, les effets ne sont pas additifs. L'effet global n'est pas égal à la somme des effets individuels. Et les modèles que l'on doit introduire ne sont donc pas linéaires : leur résolution mathématique (par exemple, la technique de réduction de variance) devient très lourde, voire impossible. On peut parfois se proposer de tester des modèles encore approximativement multilinéaires. Mais ils finissent par perdre en signification : il devient alors préférable de construire directement un modèle complexe, non linéaire cette fois-ci, de le faire simuler par l'ordinateur et d'observer le résultat de ses simulations dans diverses conditions de paramètres $^{18}$.

C'est essentiellement à cause de ce problème que la simulation sur ordinateur s'est développée, à partir des années 1970, dans les sciences à phénomènes multifactoriels et en interaction (sciences de la vie, de l'environnement, sciences sociales). Boudon avait prédit ce passage pour les sciences sociales en $1967^{19}$. En 1968, Jay Forrester écrivait également dans Principes des systèmes :

La plupart des comportements dynamiques des systèmes sociaux peuvent seulement être représentés par des modèles qui sont non linéaires et si

18 C'est l'argumentaire explicite d'André Vessereau dans Méthodes statistiques en biologie et agronomie, Paris, Lavoisier, 1988, p. 298. Une première édition de cet ouvrage remonte à 1947. Avant ceux, plus tardifs, de Jean-Pierre Benzécri puis Marc Barbut, il a été un ouvrage fondamental dans la formation en statistiques des chercheurs français en sciences sociales.

19 Dans L'analyse mathématique des faits sociaux, Raymond Boudon formulait également cette précision : "L'avantage de la simulation par rapport à la déduction réside dans le fait que l'axiomatique d'un modèle simulé n'est pas soumise à l'impératif de parcimonie. Cependant, une expérience de simulation est toujours particulière, dans la mesure où elle dépend des spécifications imposées au modèle » (op. cit., p. 408). 
complexes que des solutions mathématiques analytiques sont impossibles.

Pour de tels systèmes seul un processus de simulation utilisant une résolution numérique pas à pas est utilisable ${ }^{20}$.

Cependant, alors que pour Forrester, il s'agit d'invoquer la simulation simplement afin de résoudre numériquement des systèmes d'équations de flux non analytiquement solubles (donc par sous-symbolisation de modèles analytiques préalables), pour Boudon - et c'est encore aujourd'hui le même argument qui est invoqué par des tenants des sciences sociales computationnelles comme Nigel Gilbert et Klaus Troitzsch ${ }^{21}$, par exemple -, ce besoin se greffe sur une autre demande, opportune de son point de vue : le modèle rendu nécessaire par ce passage à la simulation se distingue du modèle statistique d'analyse précisément parce qu'il est un modèle de simulation qui se révèle être aussi un modèle causal. C'est un modèle pouvant expliquer, en des termes explicitement causaux, et non simplement reproduire ou analyser les corrélations existant entre les variables. En effet un tel modèle formel (nommé plus tard "structure causale» par Boudon) met en œuvre des relations entre symboles qui sont supposées refléter aussi les liens causaux et les interactions subsistant dans la réalité sociale. Un tel modèle vaut donc en grande partie par son pouvoir de dénoter de manière au moins partiellement iconique quelques relations choisies et que l'on suppose réellement à l'œuvre dans le monde causal.

De là vient la différence - qui demeure encore aujourd'hui entre ce que la littérature nomme souvent un "modèle de simulation » (que Boudon appelait " modèle simulé » en 1967) et une "simulation de modèle ». Si l'opération cognitive fait porter l'emphase sur le modèle, la simulation reste un simple instrument de traitement, c'est-à-dire de calcul, au service d'un modèle. Le rôle de la simulation reste ancillaire par rapport au modèle. On a alors affaire à une "simulation de modèle ". Mais si l'on fait porter l'emphase sur la simulation directe du phénomène cible, c'est le modèle qui joue ce rôle ancillaire pour la simulation : les

$20 \quad$ Jay Forrester, Principes des systèmes, Lyon, PUL, [1968] 1984, p. 310.

21 Nigel Gilbert et Klaus Troitzsch, Simulation for the Social Scientist, Philadelphia, Open University Press, 1999. 
rôles sont inversés. On produit alors un « modèle de simulation » qui sera déterminé à suivre les contraintes prioritaires de ce que l'on souhaite obtenir : la simulation du système cible. Par la suite, ce type de modèle sera dit souvent " computationnel " car ne possédant pas (à première vue) d'équivalent mathématique immédiat, c'est-à-dire de formulation formelle à la fois déductive et abréviative ${ }^{22}$.

\section{Micro-simulation}

Dans les années 1960, avec la technique de micro-simulation introduite par Guy Orcutt ${ }^{23}$ et son équipe, on assiste à l'une des premières véritables occurrences de la pratique de la simulation computationnelle pour les sciences sociales. Forrester ne fonde pas, quant à lui, l'approche computationnelle. Car, de son côté, il ne fait que transférer dans les sciences sociales une technique de calcul déjà bien développée dans les sciences de la nature : il met en œuvre des simulations numériques de modèles mathématiques continus (modèle de flux et de rétroactions continus avec équations différentielles analogues à des réseaux trophiques ou à

22 Dans son ouvrage Generative Social Science - Studies in Agent-Based Computational Modeling, Princeton University Press, 2006, Joshua Epstein rappelle cependant qu'un "modèle de simulation » sur ordinateur (y compris donc un modèle de simulation à agents) est, en principe, toujours équivalent à une approche formelle déductive. Car on peut toujours le reformuler en ce sens : l'informatique théorique est là pour le montrer. Mais Epstein ajoute l'idée essentielle que le mode de présentation (que je dirais pour ma part ici plus "iconique ") d'un modèle dit computationnel le rend " plus immédiatement intelligible » et signifiant pour un praticien des sciences sociales ( $\mathrm{p}$. xiv). Et c'est là son apport épistémique essentiel.

23 Guy Orcutt, "A New Type of Socio-Economic System ", The Review of Economics and Statistics, vol. 39, $\mathrm{n}^{\circ}$ 2, mai 1957, p. 116-123; "Simulation of Economic Systems ", The American Economic Review, vol. 50, n ${ }^{\circ} 5$, décembre 1960, p. 893-907. Même si Orcutt s'inscrit d'abord dans les suites des approches de simulation numériques par techniques de Monte-Carlo (techniques qui, selon les écoles, peuvent être interprétées - ou non - comme étant individus-centrés), dès 1960, il énonce que, de manière générale, "les techniques de simulation rendent possible l'étude effective de modèles contenant un grand nombre de composants, de variables, et de relations sous presque autant de formes que l'on puisse désirer » et surtout qu' " elles rendent les chercheurs plus complètement libres d'être guidés par des considérations relatives à l'adéquation de la représentation ", p. 900. 
des modèles environnementaux à compartiments). Sa proposition porte davantage sur le modèle que sur la simulation : elle est donc plus spéculative et conceptuelle que proprement technique et méthodologique.

La problématique socio-économique particulière d'Orcutt est bien différente et moins spéculative. En ce sens, elle sera plus féconde méthodologiquement. Elle consiste à essayer de prédire quels seraient les effets collectifs de telle ou telle politique fiscale ou sociale à partir d'une agrégation computationnelle des comportements individuels (comportements individuels d'individus - au sens propre - ou encore de foyers ou de firmes). Le principe de la micro-simulation est le suivant :

Se servir de l'ordinateur pour modéliser simultanément les deux niveaux, le niveau de l'individu et le niveau agrégé (variables macro-économiques...).

Les individus sont regroupés dans un échantillon représentatif de la population.

Dans la structure informatique, les individus sont représentés par des attributs comme le sexe, l'âge, le statut marital, le niveau d'éducation, le type d'emploi occupé, le niveau de revenus...

À chaque pas de temps, on applique aux individus de cet échantillon un modèle probabiliste de transition.

Les paramètres de ce modèle probabiliste sont dans un premier temps mathématiquement inférés à partir du modèle phénoménologique valant à l'échelle macro. Il est alors mathématiquement normal que la phase opératoire (de computation probabiliste au niveau des individus) donne ensuite lieu, dans la seconde phase de la simulation, à l'observation des mêmes valeurs de variables agrégées que le modèle macro-économique ou démographique global, modèle phénoménologique par ailleurs calibré sur le terrain. En effet, la computation rend par là de nouveau (approximativement) continu ce qu'on avait préalablement discrétisé : exception faite des opérations d'arrondis, l'information n'a donc pratiquement pas changé.

En quoi est-ce une simulation? C'est une simulation par sous-symbolisation d'un modèle agrégé préalable. Les paramètres 
des comportements des individus sont calculés à partir des paramètres disponibles du modèle agrégé. Ils ne sont donc pas évalués à partir d'une enquête de terrain au niveau des individus : ils ne prétendent donc pas au réalisme. Laction des individus n'est pas iconiquement représentée : seul l'est leur changement probable d'état. Ces individus n'ont donc d'iconique que leur individualité; pour le reste (leurs attributs), ils sont abstraits car ils ne font pas l'objet d'une enquête empirique ou compréhensive préalable. Cette simulation en est pourtant bien une, car elle pratique au minimum une sous-symbolisation. Mais cette soussymbolisation ne met pas en jeu des agents informatiques dont l'iconicité dans le comportement (le réalisme) serait également recherchée.

Ainsi, la micro-simulation peut être appelée une simulation numérique sociale : on numérise, on discrétise, mais on discrétise fictivement, cela de manière à pouvoir calculer pas à pas un comportement global de la société. L'individu y est à mi-chemin entre un symbole dénotant expressément une réalité sociale et un simple truc de calcul.

Quelle est la fonction épistémique de la micro-simulation? Quel est son apport? Elle permet une exploration de scénarios hypothétiques à partir d'un modèle de transition d'abord calibré et paramétré au niveau des individus fictifs. Cette exploration est appelée aussi une analyse en "what... if...". On peut, par exemple, tester par simulation l'effet probable d'une augmentation de la taxation sur les revenus. Pour cela, on modifie directement le modèle probabiliste (entre temps calibré) des transitions des individus en ajoutant cette taxation à chaque pas de temps. L'intérêt réside alors, dans la seconde phase, la phase d'observation de la simulation : sur l'ordinateur, on mesurera ou on observera des phénomènes de distorsion par rapport au comportement du modèle agrégé observé, quant à lui, dans la réalité.

La justification épistémologique de ce travail est la suivante : on suppose que toutes choses sont demeurées égales par ailleurs et que cette distorsion des valeurs agrégées (que l'on ne peut cette fois-ci qu'observer sur l'ordinateur - d'où la simulation - et non 
plus calculer sur le modèle agrégé) est bien un effet de cette seule augmentation de la taxation du revenu. On pratique ainsi une extrapolation à partir d'un modèle descriptif.

La séquence totale des actes cognitifs réalisés par le modélisateur ou délégués à la machine au cours d'une pratique de micro-simulation est donc la suivante : 1- modèle agrégé, 2- soussymbolisation du modèle par construction d'individus fictifs, 3- distorsion de la matrice causale fictive, 4- computation (c'està-dire resymbolisation, par computation), 5- observation, et 6conclusion. Au sens strict, la simulation n'est concernée que par les étapes 4 et 5 .

Comme on observe le résultat sur l'ordinateur, on peut dire que ce genre d'enquête sur simulation possède une dimension quasi-expérimentale ${ }^{24}$. Mais, comme l'assise théorique en est faible (car on ne décrit pas de manière réaliste ni compréhensive le comportement des agents), il y a peu de moyens de vérifier la validité de l'extrapolation et notamment l'hypothèse selon laquelle la distorsion introduite maintient " toutes choses égales par ailleurs".

\section{Approches par automates cellulaires}

La micro-simulation présente plusieurs autres limites. Non seulement, les individus sont fictifs, mais ils n'interagissent aucunement : ils ont à suivre des probabilités de transition individuelle qui sont déterminées par rapport à leurs attributs individuels. Ils n'ont donc pas de vie sociale : tout en menant un calcul pas à pas, la computation imite déjà un peu le système cible, cela à la différence de la pure simulation numérique de modèle à la Forrester, mais elle n'imite pas encore une vie sociale à proprement parler. Elle imite seulement le changement d'états d'individus fictifs qui se trouvent vivre dans une même société. Une

$\overline{24}$ Sur ce caractère quasi-expérimental des simulations, voir notamment Franck Varenne, Du modèle à la simulation informatique, op. cit., et Denis Phan et Franck Varenne (dir.), «Agent-Based Models and Simulations in Economics and Social Sciences: From Conceptual Exploration to Distinct Ways of Experimenting ", Journal of the Artificial Societies and Social Simulation (JASSS), vol. 13, n 1, 2010, http://jasss.soc.surrey.ac.uk/13/1/5.html. 
telle simulation ne permet donc pas de tester des hypothèses sur la constitution même des sociétés, sur le phénomène même de la construction de la socialité. C'est pourquoi, comme l'indiquent opportunément Rosaria Conte ${ }^{25}$ et Nigel Gilbert ${ }^{26}$, une étape supplémentaire semble devoir être franchie en matière de simulation computationnelle : il semble nécessaire de passer aux automates cellulaires.

Rappelons qu'un automate cellulaire (AC) est un réseau de cellules situé sur un maillage (quadrangulaire, triangulaire ou autre). Chacune de ces cellules est caractérisée par un état interne (une liste d'attributs) et une règle de transition de cet état. Dans un $\mathrm{AC}$ élémentaire, cette règle n'est fonction que de l'état des cellules voisines dans le maillage.

Les $\mathrm{AC}$ sont issus des considérations sur les automates de calcul telles qu'elles furent introduites par Turing, von Neumann, Ulam, puis Burks. Ils ont d'abord été utilisés en physique et en biologie computationnelles. L'intérêt de ce mode de formalisation pour les sciences sociales est double : 1- en formalisant les règles de changement d'état, il permet d'imiter de manière plus réaliste le comportement de décision et d'action de chaque agent social; 2- au contraire des micro-simulations, il prend en compte une véritable interaction entre les cellules qui représentent les agents (interactions, avec des effets de voisinage, etc.). On peut donc simuler des phénomènes d'influence ou d'imitation de proche en proche.

Un autre intérêt, théorique, éventuellement "idéologique ", des AC est qu'ils peuvent se passer d'un contrôle par un modèle valant au niveau macro. Tout peut se produire de façon bottom-up. Il n'y a pas la nécessité d'une régulation top-down du tout sur ses parties. Comme le disent Epstein et Axtell dans le

\footnotetext{
25 Rosaria Conte, "The Necessity of Intelligent Agents in Social Simulation ", dans Gérard Ballot et Gérard Weisbuch (dir.), Application of Simulation to Social Sciences, London, Hermes, 2000, p. 19-38.

26 Nigel Gilbert, "Computational Social Science - Agent-Based Social Simulation ", dans Denis Phan et Frédéric Amblard (dir.), Agent-Based Modelling and Simulation in the Social Sciences, Oxford, The Bardwell Press, GEMAS Studies in Social Analysis Series, 2007, p. 115-133.
} 
titre même de leur livre de 1996 (Growing artificial societies Social Sciences from the bottom up), il paraît envisageable de "faire pousser des sociétés artificielles du bas vers le haut", c'est-à-dire, selon eux, comme on peut supposer que les sociétés réelles ont effectivement cru.

Parmi les approches par automates cellulaires (ou apparentées), on peut ranger le modèle de Schelling ${ }^{27}$. J'en résume ici l'esprit ${ }^{28}$. La question que se pose Schelling est la suivante : l'apparition de ghettos dans les villes américaines est-elle due à un fort racisme des américains? Il fait l'hypothèse que le racisme agit sur les relations de voisinage et incite plus ou moins à déménager. Dans son modèle, il se limite à cette interaction sociale, en supposant, de plus, qu'il n'y a que deux types d'individus (deux couleurs). Schelling considère alors un échiquier dont chaque cellule peut être soit inoccupée, soit occupée par un individu d'une couleur, soit occupée par un individu de l'autre couleur. Ce schéma est équivalent à un $\mathrm{AC}$. Schelling teste plusieurs règles de tolérance : l'individu tolère telle ou telle proportion de voisins de couleurs différentes de la sienne. Si le seuil de tolérance est dépassé, l'individu se déplace : techniquement, sa cellule devient inoccupée et une cellule voisine "plus tolérable" pour lui devient occupée. À chaque pas de temps, chaque cellule applique donc des tests de tolérance et l'individu qui s'y trouve se déplace ou non.

La simulation montre (c'est-à-dire "fait voir ", " donne à observer et à mesurer ») qu'au bout d'un grand nombre d'itérations, les cellules forment des agrégats monocolores. Ils deviennent visibles au niveau macro, même quand le seuil de tolérance est élevé, seuil élevé qu'on suppose pourtant être la marque d'un racisme "faible ». Or, ces agrégats observés sur la simulation ont une allure générale semblable aux ségrégations spatiales observées sur le terrain : il y a donc iconicité à ce niveau-là aussi et non pas

\footnotetext{
27 Thomas C. Schelling, "Models of Segregation ", The American Economic Review, vol. 59, n² 2, 1969, p. 488-493.

28 Je renvoie également à la présentation et à l'analyse actualisée et critique d'Eric Daudé et Patrice Langlois, "Comparison of Three Implementations of Schelling's Spatial Segregation Model ", dans Denis Phan et Frédéric Amblard, op. cit.
} 
seulement au niveau des individus stylisés présents sur l'échiquier. Le statut épistémique de la simulation est donc différent de la micro-simulation : on a non seulement une iconicité pour l'individualité des agents sociaux (un individu est dénoté par un individu dans le programme), mais les règles d'action des agents sont choisies pour être réalistes (et donc iconiquement dénotées dans le programme) bien que fortement simplifiées. Si, de plus, l'allure du résultat de computation est elle-même réaliste $\left(3^{\mathrm{e}}\right.$ niveau d'iconicité), il semble que l'on puisse en conclure que la computation a effectivement testé un mécanisme social. Du fait de ces trois niveaux d'iconicité, il semble qu'une simulation à $\mathrm{AC}$ soit une formalisation qui conserve plus constamment et plus solidement son parallélisme avec la réalité sociale qu'un modèle mathématique : "de la simulation à la réalité, la conséquence est bonne ", croit-on alors pouvoir dire.

À tout le moins, une telle simulation montre qu'un mécanisme de ce type est au moins suffisant pour mener à de telles émergences. L'argument est donc théorique, en réalité. Il ne prouve pas directement une hypothèse mécaniste, mais il montre sa plausibilité de principe. En l'espèce, il montre qu'il n'est pas impossible que la ségrégation spatiale ne soit due quà ce facteur unique du seuil de tolérance.

\section{Approches par agents}

Les automates cellulaires, cependant, ont eux aussi des limites dans leur capacité de représentation. Les sciences sociales recourent en fait de plus en plus aux agents et aux systèmes multiagents plutôt qu'aux AC. Le concept informatique d'agent vient en grande partie de l'intelligence artificielle distribuée. Je renvoie à ce sujet à l'étude de référence de Ferber ${ }^{29}$. Gilbert résume en quatre points ce qui caractérise un agent ${ }^{30}$ :

- Autonomie. Il n'y a pas de contrôleur global qui dicte ce qu'un agent doit faire; il fait ce pour quoi il est programmé

\footnotetext{
29 Jacques Ferber, Les systèmes multi-agents, Paris, Interéditions, 1995.

30 Nigel, Gilbert, Agent-Based Models, Los Angeles, SAGE Publications, 2008, p. 21 .
} 
au vu de la situation dans laquelle il se trouve.

- Capacité sociale. Il est capable d'interagir avec les autres agents.

- Réactivité. Il est capable de réagir de manière appropriée à des stimuli venant de son environnement.

- Proactivité. Il a un but ou des buts qu'il poursuit de sa propre initiative.

Dans ce type de simulation computationnelle, l'iconicité des dimensions psychologiques mais aussi physiques de l'agent est considérablement accrue par rapport à ce qu'elle est dans les automates cellulaires. Comme le souligne Conte ${ }^{31}$, les AC sont des unités homogènes qui interagissent en suivant des règles très simples et peu réalistes. Les agents, au contraire, peuvent être très hétérogènes, c'est-à-dire différents les uns des autres. Et ils peuvent évoluer au cours de la simulation. Enfin, n'ayant pas de capacité de représentation et de délibération, les AC ne sont pas du tout proactifs.

Prenons, comme exemple, celui que nous suggère Gilbert : la simulation de l'émergence de la société (Emergence of Society ou EOS) par Jim Doran et son équipe ${ }^{32}$. Le but de cette équipe est de simuler la croissance de la complexité des institutions sociales au Paléolithique supérieur. À cette période, il y a une transition d'une société de chasseurs-cueilleurs relativement égalitaire à une société plus complexe, avec l'apparition de prises de décisions centralisées et une différenciation des rôles sociaux. L'équipe de Doran a utilisé une simulation avec des agents disposant chacun de ce qu'elle appelle un "modèle social " : ce modèle contient des croyances au sujet de l'agent lui-même et des autres agents :

Le modèle social est conçu pour enregistrer les croyances des agents à propos de l'existence de groupes d'agents et de l'identité de leurs leaders. Il y a d'autres informations qui sont associées à la croyance au sujet des leaders, par exemple la taille [du groupe]

31 Rosaria Conte, op. cit., p. 23.

32 Jim Doran et al., «The EOS Project: Modelling Upper Paleolithic Change», dans Nigel Gilbert et Jim Doran (dir.), Simulating Societies: The Computer Simulation of Social Phenomena, London, UCL Press, 1994, p. 195-221. 
de leurs suiveurs immédiats. Un agent peut très bien apparaître dans son propre modèle social comme un leader ou un suiveur. Dans ce modèle social, il y a aussi des croyances à propos des territoires. On ne demande pas que l'information contenue dans le modèle social d'un agent soit complète ni précise ${ }^{33}$.

Le modèle social a un effet sur la façon dont un agent se comporte avec les autres agents. Par exemple, s'il croit qu'il doit suivre un autre agent, il ne va pas se comporter de la même manière. Le fait qu'il finisse par croire cela est en lui-même déterminé par une règle opérant à partir d'un certain nombre d'observations de son environnement : le modèle social de chaque agent est donc évolutif.

\section{6. Évolution des agents : évolutifs et plus intelligents}

Ces travaux, auxquels il a pourtant participé, sont néanmoins critiqués par Gilbert $^{34}$. Dans l'EOS, en effet, le groupe émerge bien des actions des agents. Mais, en tant que tel, il reste une propriété implicite du modèle social des agents : il n'apparaît que comme le résultat d'un décompte d'agents suiveurs du point de vue d'un agent qui les observe. Or, Gilbert rappelle qu'une société humaine est aussi une société dans laquelle les individus réfléchissent directement sur les institutions comme telles, sur les groupes qui y émergent et y subsistent, et pas seulement sur leurs propres manières d'agir ou de modifier leurs actions par rapport à tel ou tel agent, fût-il un leader. Dans une société humaine, les éléments du niveau micro doivent donc pouvoir se représenter directement certaines propriétés qui ont émergé au niveau macro. Et ces représentations elles-mêmes, via les interprétations des agents, doivent faire retour sur l'action individuelle : ce sont des propriétés qui donnent lieu à des structurations qui structurent en retour l'action individuelle. Gilbert invoque à ce titre la théorie de la structuration de Giddens ${ }^{35}$. Mais, au sujet de cette réentrance du macro dans le micro, on pourrait également

33 Jim Doran et al., op. cit., cité par Gilbert, "Holism, Individualism and Emergent Properties ", op. cit., p. 9.

34 Nigel Gilbert, ibid., p. 9-10.

35 Anthony Giddens, La constitution de la société, Paris, PUF, [1984] 2005. 
invoquer les conceptions de Coleman ${ }^{36}$ ou encore celles de Dupuy $^{37}$, ainsi que le fait Manzo ${ }^{38}$. En sortant même du cadre de l'individualisme méthodologique, on pourrait aussi évoquer les structures structurées structurantes de la théorie de la pratique de Bourdieu ${ }^{39}$.

La critique de Gilbert revient à inciter, d'une part, à une complexification du modèle social mis en œuvre par les agents computationnels et, d'autre part, à modéliser plus correctement (c'est-à-dire de manière plus directement iconique) la sédimentation des pratiques dans des propriétés structurantes. Être membre d'un groupe suppose, en effet, aussi un type d'institutionnalisation des actions individuelles en pratiques reconnues ${ }^{40}$. Dans une direction différente de celle de Gilbert, mais de même type, Conte en appelle à une complexification (mesurée) des agents $^{41}$. Il faut, selon elle, aller vers des agents plus intelligents encore, permettant notamment de simuler l'intelligence sociale ainsi que le rôle des émotions, émotions récemment remises au premier plan en psychologie cognitive.

Issus de problématiques au départ différentes, les travaux récents de Mantzavinos ${ }^{42}$ ont également tendance à introduire des modèles mentaux complexes dans les agents formels. Ses modèles sont inspirés des travaux de psychologie cognitive évo-

\footnotetext{
36 James Coleman, Foundations of Social Theory, Cambridge, Harvard University Press, 1990.

37 Jean-Pierre Dupuy, Introduction aux sciences sociales, op. cit.

38 Gianluca Manzo, 2007, op. cit.

39 Pierre Bourdieu, Le sens pratique, Paris, Minuit, 1980. Il est intéressant de noter que les idées de Bourdieu sur l'échange et la domination peuvent être opérationnalisées par une simulation à agents : voir Jamal Alam Shah, Frank Hillebrandt et Michael Schillo, "Sociological Implications of Gift Exchange in Multiagent Systems", Journal of Artificial Societies and Social Simulation (JASSS), vol. 8, no 3, 2005, http://jasss.soc.surrey.ac.uk/8/3/5.html.

40 C'est le sens de la piste de recherche suggérée par Jacques Ferber, Leonardo Lana de Carvalho, Denis Phan et Franck Varenne, "Qu'est-ce que suivre une règle? Réflexion sur les normes et les usages ", Journées de Rochebrune 2009, article à paraître, 2010.

41 Rosaria Conte, "The Necessity of Intelligent Agents in Social Simulation ", op. cit.

42 Chrystomos Mantzavinos, Individus, institutions et marchés, Paris, PUF, 2008.
} 
lutionniste. Afin d'expliquer l'émergence des institutions et des normes, les modèles d'individus qu'il propose ne sont pas tant de représentation que de "résolution de problèmes nouveaux ». L'individu peut ainsi créer de nouvelles règles ou s'imaginer que de nouvelles règles sont à l'œuvre dans les groupes qui apparaissent de fait sous ses yeux et qu'il observe. Et le fait qu'il partage le même modèle mental créatif que les autres agents, tout en étant différent d'eux par d'autres aspects, a pour conséquence que l'agent individuel va se retrouver d'accord avec les autres agents pour cette création de règles. Or, ces règles créées/imaginées à part soi (in petto) par l'individu se révèleront a posteriori également similaires entre elles du fait que les procédures d'apprentissage (comme l'analogie, par exemple) suivies par chaque individu auront été les mêmes que - ou semblables à - celles qui sont parallèlement suivies par les autres. À l'image de la solution épistémologique proposée par le sujet transcendantal kantien, le problème de l'explication de l'émergence spontanée (non voulue par les agents) de certaines institutions ainsi que du maintien de ces institutions semble donc pouvoir être résolu par cette théorie de l'invention cognitive, invention d'abord solitaire, opérant en chacun, mais finalement toujours déjà partagée.

Pourtant, dans un tel travail, ce qu'il faut bien appeler une théorie de l'" invention simultanée " reste encore un argument essentiellement général et spéculatif. Certains diront qu'il lui manque encore de se confronter aux données de terrain. Et là, les simulations computationnelles pourraient faire l'affaire car elles offrent les médiations avec le terrain actuellement les plus souples (si pas les plus puissantes), cela du fait même de la multiplicité des niveaux d'iconicité qu'elles peuvent simultanément mobiliser. Les cadres formels qu'elles imposent sont en effet moins contraignants que ceux qui sont imposés par les traditionnels modèles mathématiques ou même que ceux de la théorie des jeux. Un certain nombre de tenants des approches computationnelles jouent précisément de cela et c'est la raison pour laquelle ils demandent aujourd'hui davantage de réalisme encore dans les agents. 


\section{Réalisme des formalismes et valeur de preuve des simulations}

Contre ce genre de demandes et contre ce qui peut apparaître une dérive illusoire vers une simulation fac-simile ${ }^{43}$, nombre d'auteurs s'élèvent cependant pour rappeler qu'il est de bonne méthode de s'en tenir à l'approche KISS caractérisée par Axelrod

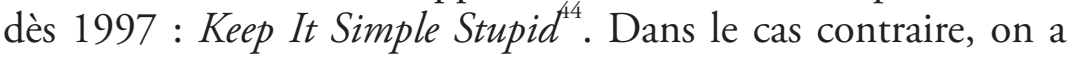
effectivement affaire à des paramètres qui ne sont pas toujours identifiables ou à des phénomènes qui ne sont pas génériques.

Cependant, cette critique porte dans les cas où l'on donne préférentiellement comme fonction épistémique à la simulation computationnelle celle d'une exploration théorique ou conceptuelle. Elle vaut donc surtout si l'on cherche des plausibilités de principe, des mécanismes généraux, ou encore si l'on cherche à mettre en évidence un résultat contre-intuitif (complexe en ce sens) se manifestant à partir d'un modèle relativement simple. La simulation du modèle de dynamique des opinions de Deffuant et son équipe ${ }^{45}$ donne une illustration de ce dernier cas. Gilbert lui-même admet que cet usage théorique reste majoritaire ${ }^{46}$, mais la tendance vers toujours plus de détail et de réalisme semble démentir cette prudence affichée.

En réalité, avec le tournant computationnel, il y a place pour toutes ces approches. Il est clair que se livrer à une exploration conceptuelle par ordinateur ou tester la plausibilité d'un méca-

43 Gilbert (Agent-Based Models, op. cit.) parle en effet dans ce cas de modèles fac-simile. Il cite le cas de l'expérimentation sociale virtuelle effectuée par Kohler et ses collègues, travail qui peut passer pour de l'archéologie expérimentale virtuelle.

44 Robert Axelrod, "Advancing the Art of Simulation in the Social Sciences ", dans Rosaria Conte, Rainer Hegselmann et Pietro Terna (dir.), Simulating Social Phenomena, Berlin, Springer-Verlag, 1997, p. 21-40.

45 Guillaume Deffuant, Frédéric Amblard, Gérard Weisbuch et Thierry Faure, "How Can Extremism Prevail? A Study Based on the Relative Agreement Interaction Model ", Journal of Artificial Societies and Social Simulation (JASSS), vol. 5, no 4, 2002, http://jasss.soc.surrey.ac.uk/5/4/1.html.

46 C'est également le point de vue le plus représenté dans John H. Miller et Scott E. Page, Complex Adaptive Systems - An Introduction to Computational Models of Social Life, Princeton (NJ), Princeton University Press, 2007. Voir notamment le chapitre 5 intitulé "Computation as theory ". 
nisme général n'est pas la même chose que de constituer un laboratoire social virtuel. De quoi la différence dépend-elle? Elle dépend essentiellement du type symbolique que l'on attribue aux symboles que l'on fait traiter par l'ordinateur : on trouve les détails techniques de ces différences dans l'article de Denis Phan et Franck Varenne ${ }^{47}$.

En bref, ici, on peut dire que, dans une simulation computationnelle, chaque niveau de système de symboles entretient des rapports, d'une part, avec les autres niveaux de symboles qui sont internes à la simulation (par exemple des rapports de soussymbolisation, ou d'exemplification) et, d'autre part, avec des choses ou d'autres types de symboles qui sont reconnus par une théorie, un savoir scientifique ou encore un sens commun comme faisant partie du monde extérieur à la simulation. S'il concerne uniquement des rapports internes entre les niveaux de symboles, un rapport d'iconicité est purement relatif à un changement de niveaux. Par exemple, les symboles élémentaires intervenant dans la simulation numérique d'un fluide (en physique des fluides) ou dans la simulation numérique d'un modèle cybernétique de Forrester (en sciences sociales) manifestent une iconicité interne relative. Ils sont iconiques relativement aux variables agrégées qu'ils sous-symbolisent. Mais ils ne sont pas iconiques relativement au monde extérieur : c'est ce que l'on veut exprimer quand on dit qu'ils ne sont pas réalistes. Leur pouvoir dénotationnel (qui est ici de ne "rien " dénoter) a besoin du passage par le langage conventionnel pour être effectif : on dit d'ailleurs que ce sont des éléments finis "fictifs" ou des symboles dénotants "sans dénotation ». Ce fait qu'ils ne dénotent " rien " ne peut pas se montrer, mais cela se dit, par passage au langage, à autre niveau symbolique, donc. Or, il ne faut pas confondre cette iconicité relative et interne avec l'iconicité externe.

Le tournant computationnel a pour principal effet de brouiller les frontières conceptuelles, frontières qu'il faut d'abord avoir aperçues pour comprendre et saisir les limites mêmes de ce tournant. Car il témoigne justement de l'ambition de faire en

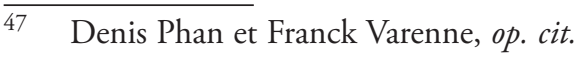


sorte que certains symboles opératoires, qui manifestent donc déjà une iconicité relative interne, soient en même temps dotés d'une certaine iconicité externe. La micro-simulation dote ainsi les symboles des individus du double statut de " truc de calcul ", d'une part, et de symboles dénotants plus ou moins bijectivement des agents sociaux, d'autre part. Le fait qu'ils dénotent chacun, par une relation d'un à un, un individu réel (certes fortement stylisé) est bien ce qui fait la nouveauté de la méthode de microsimulation. Une autre iconicité entre en jeu, ici, et semble ensuite pouvoir frauduleusement se confondre avec la première. Or, les deux restent différentes en nature : on peut discuter du bien fondé d'une iconicité externe, mais pas de celle d'une iconicité interne. L'autorité de l'une ne peut, sans validation niveau par niveau, hériter de l'autorité de l'autre. On peut, en effet, douter du réalisme d'un formalisme puisqu'on peut même douter de la réalité et de la consistance de telle ou telle entité externe postulée par un savoir plus ou moins immédiat. Mais le fait qu'un système de symbole en sous-symbolise un autre est quelque chose qui est dû au recours à l'ordinateur et à son fonctionnement computationnel.

Comme on l'a vu, le passage aux automates cellulaires permet d'accroître le nombre de dimensions d'iconicité externe : à côté de l'iconicité individu/individu, le voisinage réel des agents est rendu par un voisinage dans la grille. Comme on a, dans ce cas, les résultats d'une interaction plus que ceux d'une déduction formelle ou d'un calcul analytique approché, ce qui devient alors important à tester est la robustesse des résultats de simulations : $\mathrm{a}$-t-on un résultat de même allure pour des paramètres légèrement différents? Ensuite, cette allure ressemble-t-elle à ce qu'on observe sur le terrain?

Enfin, le passage des AC aux simulations à agents permet d' " humaniser " encore un peu plus les structures informatiques et d'augmenter le nombre des dimensions d'iconicité externe. C'est une demande forte actuellement car, par ailleurs, plus une simulation computationnelle est riche, formellement souple et virtuellement détaillée, plus elle peut être aisément couplée avec 
d'autres modèles ou avec des bases de données comme des systèmes d'information géographique : l'actuelle archéologie virtuelle de Kohler et son équipe ${ }^{48}$ permet de conduire des expériences qui prolongent les techniques de l'archéologie expérimentale classique. Ces simulations détaillées mais validées à chaque ajout de sous-modèles (validées dans leurs dimensions d'iconicité externe) ont aussi un aspect incrémental indéniable comme le précise Kohler lui-même : chaque chercheur peut proposer d'enrichir la simulation avec son sous-modèle ou sa proposition descriptive ou explicative (c'est-à-dire de mécanisme) ${ }^{49}$. J'ajouterais ici que le dialogue entre différentes spécialités ne confine pas ainsi immédiatement à une dialectique conflictuelle où tel ou tel réductionnisme serait en jeu. À cet égard, les approches dialectiques, en sciences sociales, peuvent rétrospectivement être vues comme l'effet d'une rationalisation des limites techniques de certains formalismes hégémoniques ayant régné à l'ère précomputationnelle, ainsi que des conceptualisations qui leur étaient associées.

\section{Conclusion}

Rétrospectivement, les principaux apports des méthodes de simulation computationnelle peuvent donc être résumés ainsi ${ }^{50}$.

1- Elles permettent de formaliser le caractère dynamique des phénomènes sociaux, leur historicité ${ }^{51}$.

2- Elles permettent de modéliser les effets en retour des institutions émergeantes et des structurations sociales sur l'action individuelle.

3- La spatialité (ou, plus généralement, le caractère réticulaire,

48 Tim Kohler, George Gumerman et Robert Reynolds, «Simulating Ancient Societies ", Scientific American, juillet 2005, p. 76-84.

49 Ibid., p. 82.

50 Voir également une liste proche dans John H. Miller et Scott E. Page, op. cit., p. 78-89.

51 Nigel Gilbert, "Holism, Individualism and Emergent Properties ", dans Rainer Hegselmann, Ulrich Mueller et Klaus Troitzsch (dir.), Modelling and Simulation in the Social Sciences from the Philosophy of Science Point of View, Dordrecht, Kluwer, 1996, p. 1-12 et Nigel Gilbert, Agent-Based Models, op. cit. 
c'est-à-dire le fait d'interagir dans des réseaux) des phénomènes sociaux n'est plus opposée à leur temporalité : du fait de la possibilité du traitement parallèle de processus séquentiels et interactifs, on n'a pas à choisir entre la représentation d'interactions spatiales et la représentation d'évolutions dans le temps.

4- L'approche par agents permet de coupler différents types de modèles et différents types de formalisation mathématiquement incompatibles ${ }^{52}$. La simulation computationnelle favorise ainsi l'interdisciplinarité entre sciences sociales (psychologie / sociologie / économie / archéologie / géographie, etc.) et entre sciences sociales et sciences de la nature (sociologie / écologie / géographie), cela tout en permettant d'éviter le réductionnisme, c'està-dire le fait de réduire une discipline scientifique à une autre ou, plus précisément, de réduire les objets ou les lois reconnus par une science à ceux reconnus par une autre. Ce réductionnisme n'est pas toujours évité toutefois : il suffit de voir la tendance moniste d'une certaine sociobiologie computationnelle ou encore de ce darwinisme multi-échelle et transdisciplinaire issu d'une généralisation un peu rapide des approches de psychologie cognitive évolutionnaire.

Cela ne fait pas pour autant des approches computationnelles le fondement d'une unité pour les sciences sociales. Car avec une telle "science sociale générative " (au sens d'Epstein et Axtell), pour laquelle on explique un phénomène social en le faisant " croître " dans une simulation, on peut parfois expliquer et prédire (ou rétrodire) à la fois. Et cela est assez nouveau pour les sciences sociales. Mais on le fait toutefois sans comprendre : on ne fait voir par là aucune unité conceptuelle ou théorique. Par conséquent, cette approche reste bien seulement une méthodologie : on n'obtient par là aucune nouvelle unité (qui serait une unité des objets, des lois, voire des concepts) des sciences sociales même si ces simulations sont souvent pluridisciplinaires. Il faut donc rejeter la thèse forte et s'en tenir à la thèse moyenne.

Les simulations computationnelles proposent en réalité - et

52 Voir une étude de cas en biologie computationnelle dans Franck Varenne, Du modèle à la simulation informatique, op. cit. 
au minimum - une nouvelle méthodologie aux sciences sociales. Cette méthodologie est inédite et puissante, il est vrai, car diversement adaptable à différentes disciplines des sciences sociales. De plus, elle favorise deux développements d'apparence contradictoires, mais en fait complémentaires : 1- un développement des sciences sociales virtuelles, fondées sur des expérimentations virtuelles, de type fac-simile, expérimentations crédibles pour peu que les problèmes difficiles de calibration et de validation y soient rigoureusement traités, souvent au cas par cas; 2- une extension symétrique du domaine conceptuel de l'explication sociale et l'entrée de fait dans l'ère d'un «individualisme méthodologique complexe".

Dans cette dernière perspective, Manzo ${ }^{53}$ souhaiterait que soit possible pour la sociologie ce qui l'est déjà en partie pour l'archéologie : l'association de la prédiction (rétrodiction) et de l'explication. Pour lui, la simulation par agents informatiques est désormais un outil innovant indispensable en sociologie dès lors qu'elle permet notamment l'explication simultanée des rapports ascendants micro $\rightarrow$ macro comme celle des rapports descendants macro $\rightarrow$ micro. Voici pourtant ce que souhaite plus précisément Manzo et qui fait l'objet de ses travaux actuels :

Par rapport à une conception des « modèles informatiques " comme de "purs laboratoires virtuels " ${ }^{4}$, une alliance, en aval, entre la technique statistique descriptive et des " modèles générateurs " à visée explicative animés par simulation pourrait ainsi satisfaire, du moins en partie, l'exigence ressentie de plus en plus fortement d'une meilleure " validation " des " modèles simulés "

53 Gianluca Manzo, " Progrès et "urgence” de la modélisation en sociologie. Du concept de "modèle générateur" et de sa mise en œuvre ", op. cit.

54 De tels " laboratoires " n'en étant pas vraiment en fait, dans son esprit (et à juste raison : voir le point de vue déjà cité de Gilbert à ce sujet) puisqu’ils valent surtout pour l'exploration conceptuelle et non pour une véritable expérimentation déléguée à la machine. Ce genre d'exploration conceptuelle reste donc pour lui encore trop détaché des données de terrain. Remarquons pourtant que ce qui est, en effet, encore le cas en sociologie computationnelle (les approches computationnelles y restent à usage majoritairement théorique et d'exploration conceptuelle) l'est peut-être déjà moins en géographie ou en archéologie computationnelles.

55 Gianluca Manzo, "Progrès et "urgence” de la modélisation en sociologie. Du 
Les travaux récents de Manzo sur les mécanismes générateurs de la stratification éducative ${ }^{56}$ sont une première tentative rigoureuse d'appliquer une méthodologie mixte qui pourrait convenir assez largement aux sciences sociales quantitatives, dès lors qu' elle combine l'approche statistique d'analyse de données, dont nous avons rappelé l'importance, et l'approche générative et explicative, par simulations computationnelles. Seul l'avenir pourra dire s'il s'agit là d'une piste féconde, voire d'une nouvelle orthodoxie méthodologique. Toutefois, il serait tout à fait prématuré d'en conclure l'idée que, sur cette base-là, une unité des sciences sociales pourrait bientôt se constituer.

\section{Bibliographie}

Allaire, Grégoire, Analyse numérique et optimisation, Paris, Éditions de l'École Polytechnique, 2007.

Axelrod, Robert, "Advancing the Art of Simulation in the Social Sciences ", dans Rosaria Conte, Rainer Hegselmann et Pietro Terna (dir.), Simulating Social Phenomena, Berlin, Springer-Verlag, 1997, p. $21-40$.

Axelrod, Robert, Comment réussir dans un monde d'égoïstes?, Paris, Odile Jacob, 2006, traduction de The Evolution of Cooperation, New York, Basic Books [1 $1^{\text {re }}$ édition : 1984].

Ballot, Gérard et Gérard Weisbuch (dir.), Application of Simulation to Social Sciences, London, Hermes, 2000.

Boudon, Raymond, L'analyse mathématique des faits sociaux, Paris, Plon, 1967.

Boudon, Raymond, Essais sur la théorie générale de la rationalité - Action sociale et sens commun, Paris, PUF, 2007.

Boudon, Raymond, La rationalité, Paris, PUF, coll. "Que sais-je?", 2009. 
Boudon, Raymond et Renaud Fillieule, Les méthodes en sociologie, Paris, PUF, coll. "Que sais-je? », [1969] 2004.

Bourdieu, Pierre, Le sens pratique, Paris, Minuit, 1980.

Coleman, James, Foundations of Social Theory, Cambridge, Harvard University Press, 1990.

Conte, Rosaria, "The Necessity of Intelligent Agents in Social Simulation ", dans Gérard Ballot et Gérard Weisbuch (dir.), Application of Simulation to Social Sciences, London, Hermes, 2000, p. 19-38.

Daudé, Eric et Patrice Langlois, "Comparison of Three Implementations of Schelling's Spatial Segregation Model », dans Denis Phan et Frédéric Amblard (dir.), Agent-Based Modelling and Simulation in the Social and Human Sciences, Oxford, The Bardwell Press, GEMAS Studies in Social Analysis Series, 2007, p. 295-326.

Deffuant, Guillaume, Frédéric Amblard, Gérard Weisbuch et Thierry Faure, "How Can Extremism Prevail? A Study Based on the Relative Agreement Interaction Model ", Journal of Artificial Societies and Social Simulation (JASSS), vol. 5, $\mathrm{n}^{\circ}$ 4, 2002, http://jasss.soc.surrey.ac. uk/5/4/1.html.

Doran, Jim, Mike Palmer, Nigel Gilbert et Paul Mellars, "The EOS Project: Modelling Upper Paleolithic Change ", dans Nigel Gilbert et Jim Doran (dir.), Simulating Societies: The Computer Simulation of Social Phenomena, London, UCL Press, 1994, p. 195-221.

Dupuy, Jean-Pierre, Introduction aux sciences sociales, Paris, Ellipses, 1992.

Epstein, Joshua, Generative Social Science - Studies in Agent-Based Computational Modeling, Princeton, Princeton University Press, 2006.

Epstein, Joshua et Robert Axtell, Growing Artificial Societies - Social Science from the Bottom Up, Cambridge, MIT Press, 1996.

Ferber, Jacques, Les systèmes multi-agents, Paris, Interéditions, 1995.

Ferber, Jacques, Leonardo Lana de Carvalho, Denis Phan et Franck Varenne, "Qu'est-ce que suivre une règle? Réflexion sur les normes et les usages ", Journées de Rochebrune 2009, article à paraittre, 2010.

Forrester, Jay, Principes des systèmes, Lyon, PUL, [1968] 1984.

Friedman, Milton, Essays on Positive Economics, Chicago, University of Chicago Press, 1953.

Giddens, Anthony, La constitution de la société, Paris, PUF, [1984] 2005.

Gilbert, Nigel, "Holism, Individualism and Emergent Properties ", dans Rainer Hegselmann, Ulrich Mueller et Klaus Troitzsch (dir.), Modelling and Simulation in the Social Sciences from the Philosophy of Science Point 
of View, Dordrecht, Kluwer, 1996, p. 1-12.

Gilbert, Nigel, "Computational Social Science - Agent-Based Social Simulation ", dans Denis Phan et Frédéric Amblard (dir.), Agent-Based Modelling and Simulation in the Social and Human Sciences, Oxford, The Bardwell Press, GEMAS Studies in Social Analysis Series, 2007, p. 115-133.

Gilbert, Nigel, Agent-Based Models, Los Angeles, SAGE Publications, 2008.

Gilbert, Nigel et Klaus Troitzsch, Simulation for the Social Scientist, Philadelphia, Open University Press, 1999.

Heath, Brian, Raymond Hill et Frank Ciarallo, «A Survey of Agent-Based Modeling Practices (January 1998 to July 2008) ", Journal of Artificial Societies and Social Simulation, vol. 12, n ${ }^{\circ} 4,2009$, http://jasss.soc. surrey.ac.uk/12/4/9.html.

Hegselmann, Rainer, Ulrich Mueller et Klaus Troitzsch, Modelling and Simulation in the Social Sciences from the Philosophy of Science Point of View, Dordrecht, Kluwer, 1996.

Humphreys, Paul, Extending Ourselves: Computational Science, Empiricism, and Scientific Method, Oxford, Oxford University Press, 2004.

Kohler, Tim, George Gumerman et Robert Reynolds, «Simulating Ancient Societies ", Scientific American, juillet 2005, p. 76-84.

Livet, Pierre, "Towards an Epistemology of Multi-Agent Simulations in Social Sciences ", dans Denis Phan et Frédéric Amblard (dir.), AgentBased Modelling and Simulation in the Social and Human Sciences, Oxford, The Bardwell Press, GEMAS Studies in Social Analysis Series, 2007, p. 169-193.

Mantzavinos, Chrystomos, Individus, institutions et marchés, Paris, PUF, 2008.

Manzo, Gianluca, "Progrès et "urgence" de la modélisation en sociologie. Du concept de "modèle générateur" et de sa mise en œuvre ", Année sociologie, vol. $57, \mathrm{n}^{\circ} 1,2007$, p. 13-61.

Manzo, Gianluca, La spirale des inégalités, Paris, Presses universitaires de Paris-Sorbonne, 2009.

Michalewicz, Marek, Plants to Ecosystems. Advances in Computational Life Sciences, Collingwood (Australia), CSIRO Publishing, 1997.

Miller, John H. et Scott E. Page, Complex Adaptive Systems - An Introduction to Computational Models of Social Life, Princeton (NJ), Princeton University Press, 2007.

Minsky, Marvin, "Matter, Mind and Models ", Proceedings of IFIP Congress, 1965, p. 45-49. 
Modélisation mathématique et simulation numérique, Les Ulis, EDP Sciences, 1999.

Morgan, Mary et Margaret Morrison (dir.), Models as Mediators, Cambridge, Cambridge University Press, 1999.

Ören, Tüncer I., " Toward the Body of Knowledge of Modeling and Simulation ", Interservice/Industry Training, Simulation, and Education Conference (I/ITSEC), Orlando, Florida, 2005, p. 1-19.

Orcutt, Guy, "A New Type of Socio-Economic System ", The Review of Economics and Statistics, vol. 39, n' 2, mai 1957, p. 116-123.

Orcutt, Guy, "Simulation of Economic Systems ", The American Economic Review, vol. 50, n 5, décembre 1960, p. 893-907.

Phan, Denis et Frédéric Amblard (dir.), Agent-Based Modelling and Simulation in the Social and Human Sciences, Oxford, The Bardwell Press, GEMAS Studies in Social Analysis Series, 2007.

Phan, Denis et Franck Varenne, "Agent-Based Models and Simulations in Economics and Social Sciences: From Conceptual Exploration to Distinct Ways of Experimenting ", Journal of the Artificial Societies and Social Simulation (JASSS), vol. 13, n ${ }^{\circ}$ 1, 2010, http://jasss.soc.surrey. ac.uk/13/1/5.html.

Schelling, Thomas C., "Models of Segregation ", The American Economic Review, vol. 59, $\mathrm{n}^{\circ}$ 2, 1969, p. 488-493.

Shah, Jamal Alam, Frank Hillebrandt et Michael Schillo, "Sociological Implications of Gift Exchange in Multiagent Systems ", Journal of Artificial Societies and Social Simulation (JASSS), vol. 8, n 3, 2005, http://jasss.soc.surrey.ac.uk/8/3/5.html.

Smolensky, Paul, "On the Proper Treatment of Connectionism ", The Behavioural and Brain Sciences, vol. 11, 1988, p. 1-74.

Varenne, Franck, "La simulation informatique face à la méthode des modèles ", Natures Sciences Sociétés, vol. 11, no 1, 2003, p. 16-28.

Varenne, Franck, Du modèle à la simulation informatique, Paris, Vrin, 2007.

Varenne, Franck, Qu'est-ce que l'informatique?, Paris, Vrin, 2009.

Varenne, Franck, "Framework for M\&S with Agents in regard to Agent Simulations in Social Sciences: Emulation and Simulation ", dans Alexandre Muzy, David R.C. Hill et Bernard P. Zeigler (dir.), Complex Systems: Activity-Based Modeling and Simulation, Corte, Corsica, 2010, p. 49-80.

Varenne, Franck, «Épistémologie des modèles et des simulations - Tour d'horizon et tendances ", dans Jean-Michel Lévy (dir.), Les modèles, possibilités et limites : jusqu'où va le réel?, Paris, EDP Sciences, à 
paraitre en 2010.

Varenne, Franck, "Modèles et simulations : pluriformaliser, simuler, remathématiser ", dans Jean-Jacques Kupiec, Guillaume Lecointre, Marc Silberstein et Franck Varenne (dir.), Modèles Simulations Systèmes, Paris, Syllepse, 2008, p. 153-180.

Vessereau, André, Méthodes statistiques en biologie et agronomie, Paris, Lavoisier, 1988.

Walliser, Bernard, "Les modèles de l'économie cognitive ", dans JeanJacques Kupiec, Guillaume Lecointre, Marc Silberstein et Franck Varenne (dir.), Modèles simulations systèmes, Paris, Syllepse, 2008, p. 183-199.

Yilmaz, Levent, Tüncer I. Ören et Nasser Ghassem-Aghaee, "Intelligent Agents, Simulation, and Gaming ", Simulation \& Gaming, vol. 37, $\mathrm{n}^{\circ} 3$, septembre 2006, p. 339-349. 